\title{
Photogrammetry Measurements During a Tanking Test on the Space Shuttle External Tank, ET-137
}

\author{
Justin Littell - ATK Space Systems, NASA Langley Research Center, 12 W. Bush Rd MS 495, Hampton VA 23681. \\ Justin.D.Littell@nasa.gov
}

Tim Schmidt, John Tyson - Trilion Quality Systems, 500 Davis Drive St 200, Plymouth Meeting PA 19462. Schmidt@trilion.com,Tyson@trilion.com

Stan Oliver - EV31, NASA Marshall Space Flight Center, Huntsville, AL 35812. Stanley.T.Oliver@nasa.gov

Matt Melis, Charles Ruggeri, Duane Revilock - NASA Glenn Research Center, 21000 Brookpark Rd MS 49-8, Cleveland OH 44135.

Matthew.E.Melis@nasa.gov, Charles.R.Ruggeri@nasa.gov,Revilock@nasa.gov

\begin{abstract}
On November 5, 2010, a significant foam liberation threat was observed as the Space Shuttle STS-133 launch effort was scrubbed because of a hydrogen leak at the ground umbilical carrier plate. Further investigation revealed the presence of multiple cracks at the tops of stringers in the intertank region of the Space Shuttle External Tank. As part of an instrumented tanking test conducted on December 17, 2010, a three dimensional digital image correlation photogrammetry system was used to measure radial deflections and overall deformations of a section of the intertank region.
\end{abstract}

This paper will describe the experimental challenges that were overcome in order to implement the photogrammetry measurements for the tanking test in support of STS-133. The technique consisted of configuring and installing two pairs of custom stereo camera bars containing calibrated cameras on the $215-\mathrm{ft}$ level of the fixed service structure of Launch Pad 39-A. The cameras were remotely operated from the Launch Control Center 3.5 miles away during the 8 hour duration test, which began before sunrise and lasted through sunset.

The complete deformation time history was successfully computed from the acquired images and would prove to play a crucial role in the computer modeling validation efforts supporting the successful completion of the root cause analysis of the cracked stringer problem by the Space Shuttle Program. The resulting data generated included full field fringe plots, data extraction time history analysis, section line spatial analyses and differential stringer peak-valley motion. Some of the sample results are included with discussion. The resulting data showed that new stringer crack formation did not occur for the panel examined, and that large amounts of displacement in the external tank occurred because of the loads derived from its filling. The measurements acquired were also used to validate computer modeling efforts completed by NASA Marshall Space Flight Center (MSFC).

\section{Background}

Space Transportation System 133 (STS-133) was the $133^{\text {rd }}$ mission in the Space Shuttle Program and the final mission for the Discovery orbiter. Discovery carried a Multipurpose Logistics Module (MPLM) "Leonardo", and an ExPRESS ${ }^{1}$ logistics carrier (ELC) to the International Space Station [1]. STS-133 was scheduled to lift off from NASA Kennedy Space Center (KSC),

1 - Expedite the processing of experiments to the $\underline{\text { ppace }} \underline{\text { Station }}$

The use of trademarks or names of manufacturers in this report is for accurate reporting and does not constitute an official endorsement, either expressed or implied, of such products or manufacturers by the National Aeronautics and Space Administration 
Launch Pad 39-A, on September 16, 2010. A series of scrubs pushed the launch back to November 5, 2010. On November 5,2010 , the launch was scrubbed because of leaks in the ground umbilical carrier plate (GUCP), which occurred during the filling process of the external tank (ET), ET-137. During the post-scrub walk down inspection, cracks were discovered in the thermal protection system (TPS) foam insulating the flange connecting the liquid oxygen (LO2) tank and the intertank near the top of the ET on Panel 2. These cracks were unrelated to the GUCP leak issue.

The intertank is an unpressurized cylinder which connects the liquid hydrogen tank (LH2) to the LO2 tank. It connects the two tanks via bolting flange rings in both its forward and aft locations. It is comprised of eight panels, six of which are thin skins with bolted on stiffeners. In some locations, additional panels, called doublers, are added to the thin skin to provide additional support. The setup of the ET can be seen in Figure 1, along with the location where the TPS cracks developed.

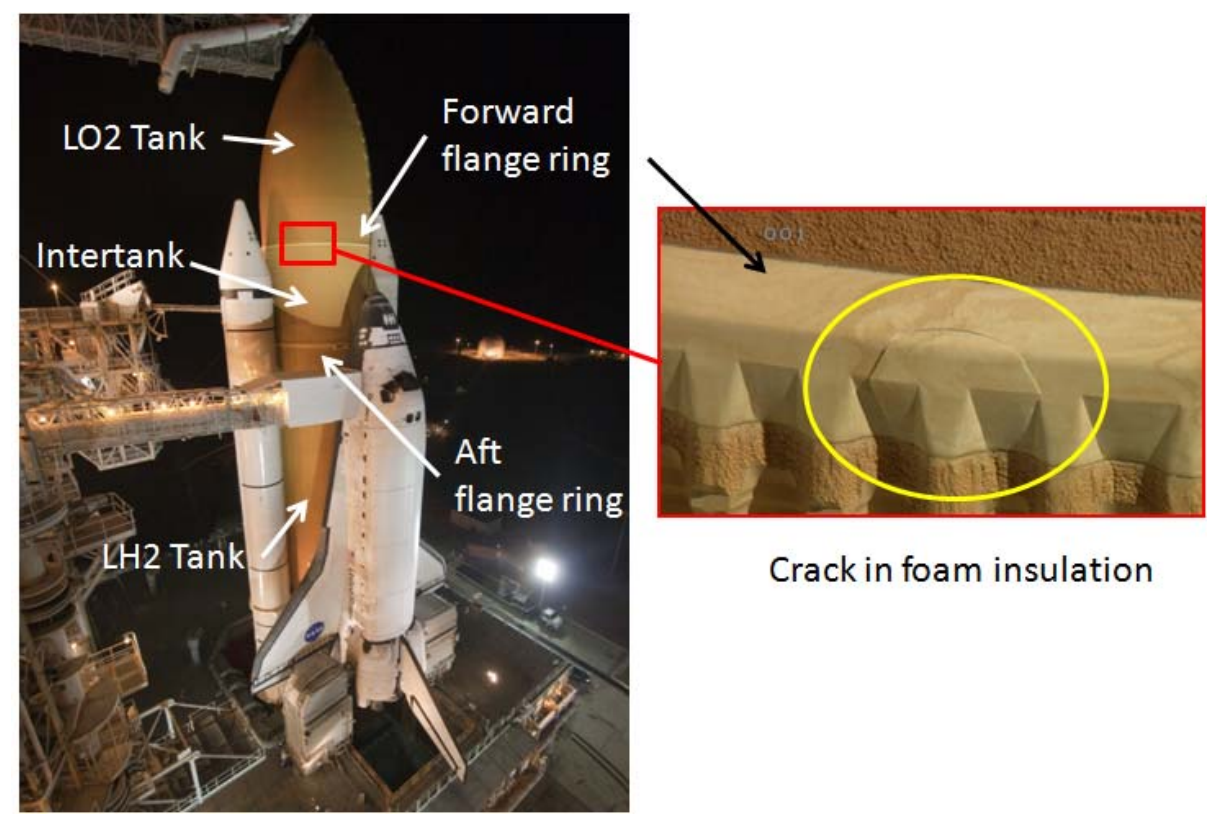

Fig. 1 Cracks in the ET foam at the LO2 flange on Panel 2 due to stringer cracking

Closer inspections indicated that the TPS cracks initiated from an area where the riveted hat stiffeners (stringers) mounted to the ET Intertank, providing additional structural support to the Intertank near the LO2/intertank interface. Inspections of the stringers necessitated cutting the TPS foam away and revealed that cracks were present in more of the aluminum stringers in regions outside the one highlighted in Figure 1. The issue was mitigated by removing the cracked portions of the original stringers and replacing with new stringers and z-doublers. The remaining un-cracked stringers received a small reinforcement on their feet [2]. New TPS foam was reapplied to the repaired region to provide insulation for the ET. Figure 2 shows a final repaired Panel 2 region. 


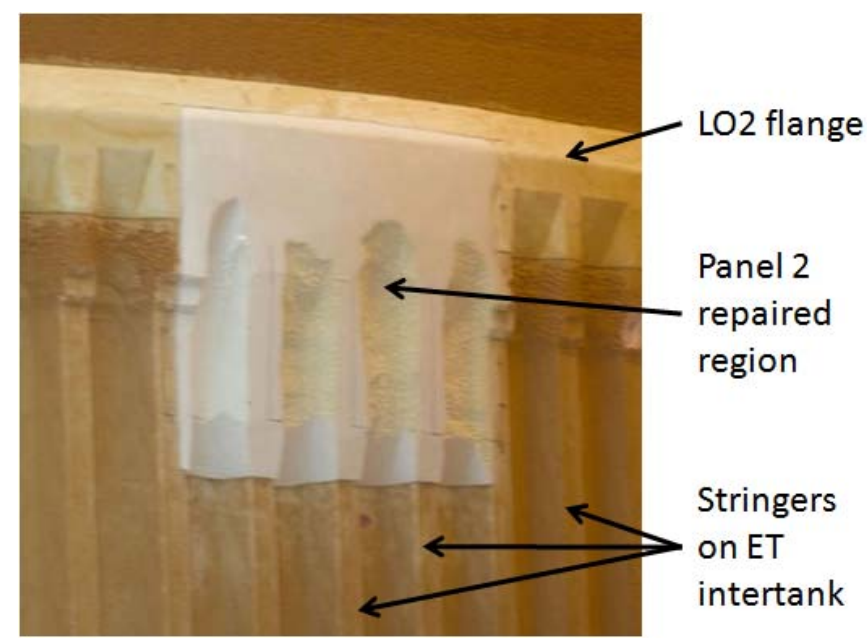

Fig. 2 A repaired region of the LO2 Flange on ET Panel 2

The Space Shuttle Program concluded that a comprehensive root cause analysis for the stringer cracks would be necessary, prior to flying the STS-133 mission, to insure flight safety for the vehicle. This investigation would require months of testing and analysis, and would include a tanking test of the external tank, ET-137, with an extensive array of strain gages and thermocouples added in an attempt to characterize the thermal and deformation state of the tank under pre-launch loads. The tanking test, lasting nearly 8 hours, effectively simulated all of the procedural steps in the launch countdown process including a complete fill, pressurization and drain with over 1.5 million pounds of the liquid oxygen and liquid hydrogen propellants.

The External Tank Project Office inquired into the feasibility of using stereo digital image correlation techniques to capture full-field deformations of the tank external surface at two separate locations during the tanking test, a measurement which has never been attempted previously. As a background, stereo digital image correlation is the acquisition of engineering data from digital stereo image pairs for the determination of object shape and deformation. The typical procedure involves the application of a dot or speckle pattern onto an object and tracking the motion of the applied pattern as loading occurs. For three dimensional digital image correlation, at least two cameras are needed such that the third, out-of-plane direction can be triangulated between camera locations. The digital image correlation that was used for the tanking test was a commercial system which had previously been used to validate LS-DYNA models of foam impact on the Orbiter wing reinforced carbon-carbon panels during the Columbia Return to Flight program [3-5]. Three dimensional digital image correlation has been successfully used to capture motion in a wide range of research areas from material characterization tests [6-9] to structural tests [10-12], to biomechanics [13-16].

The area of interest at both of these locations would include stringers and the flange structure joining the intertank to the LO2 tank. A rapid assessment from a NASA photogrammetry team and industry consultants established that it was reasonable to use digital image correlation techniques for the test, given enough time to assemble and set up the equipment. As a consequence, the photogrammetry team was assembled and tasked to move forward supporting the tanking test.

\section{Test Methodology}

After a walk down of Launch Pad 39-A conducted on December 6, 2010, photogrammetry engineers decided on a plan of action for two independent views requiring two camera systems. The first would focus on Panel 2 on the repaired region, while the second would focus on Panel 6, a similar, non-repaired panel that would serve as a baseline. 


\section{Final Camera Positions}

The Panel 6 camera pair was mounted on the railing of the GUCP walkway located on the 215-ft level of the fixed service structure (FSS) and aimed at ET Panel 6, focusing on the region near the LO2 flange. This particular region was chosen because it allowed technician and engineer access to both the cameras and the ET via the GUCP walkway, and also because Panel 6 was structurally similar to Panel 2 in terms of geometry, including similar skin thickness and doubler regions. A pair of Vision Research Phantom v10.1 4 megapixel (MP) cameras was attached onto a 13 foot long, vertically mounted 6-in- by 6-in- extruded aluminum beam at a base separation of approximately 7 feet. They were enclosed in nitrogen gas purged bags to meet the safety requirements and mitigate the risk for a spark, fire or explosion during the test. The aluminum beam was covered in a thermal protection blanket to minimize thermal expansion due to temperature change throughout the day. The thermal expansion of the beam was a major concern for the integrity of the calibration of the cameras. This issue will be discussed further in the Calibration Procedures section. Figure 3 shows the Panel 6 test setup.

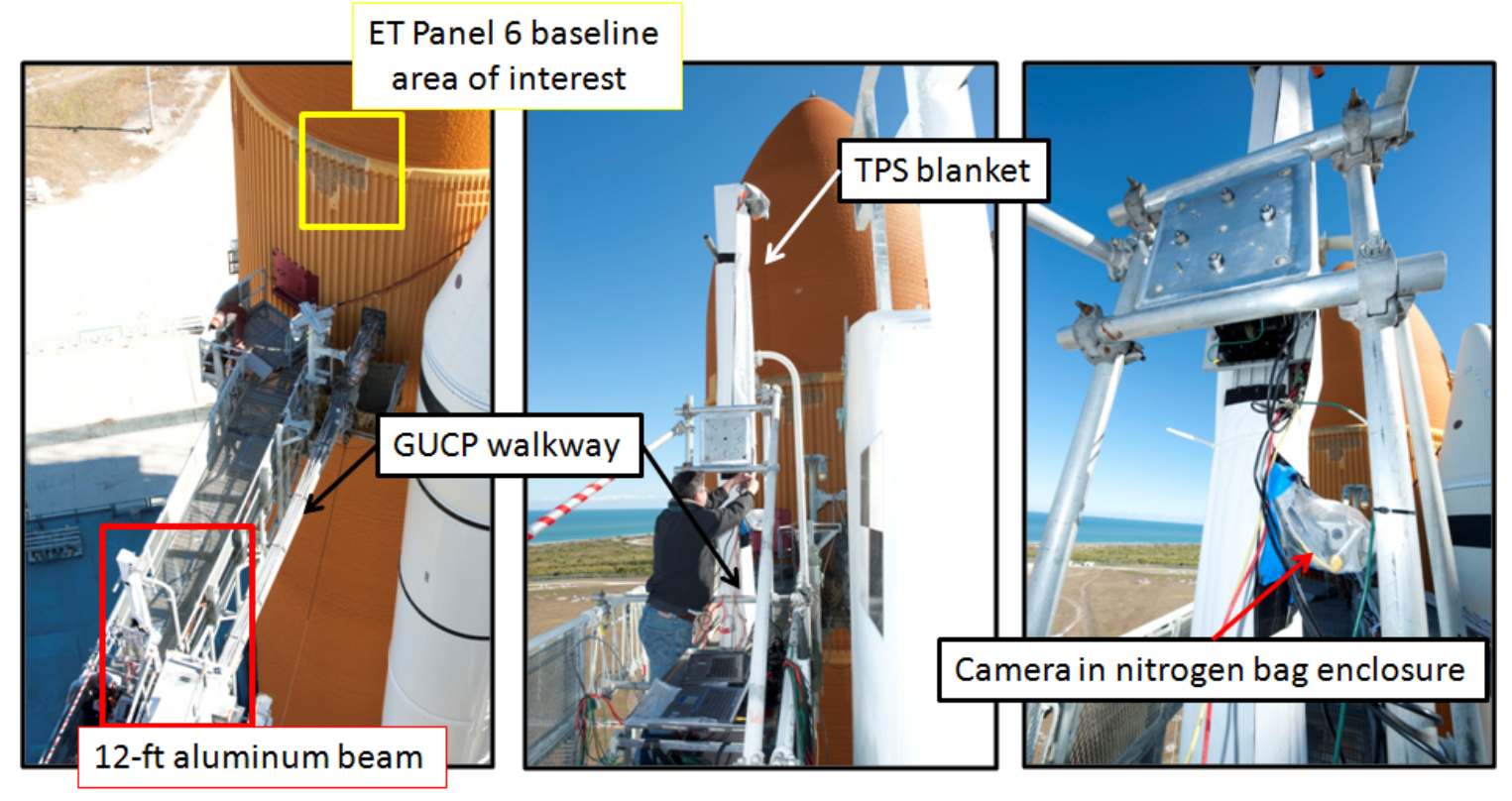

Fig. 3 Details and camera setup for ET Panel 6

A second camera pair was constrained to be used on the repaired region on ET Panel 2. The first option was to use the removable service structure (RSS) for the mounting of the camera pair because the RSS was very close to the Panel 2 region while also having ample room to setup and operate the camera equipment. However, the tanking test required that the RSS be in the open position in order to accurately simulate the launch countdown, which made it impractical to use. With the removable service structure rotated into the open position and therefore unusable, the closest available mounting location was the walkway which connected the FSS to the RSS. This walkway was located approximately 60 feet away from the ET. The $60-\mathrm{ft}$ distance constrained the camera base separation to be between $20 \mathrm{ft}$ and $30 \mathrm{ft}$. As such, Baumer $5 \mathrm{MP}$ cameras were mounted on a custom 20-ft long, 6-in diameter woven composite hollow beam. A composite beam was chosen for the beam as alternative aluminum or steel beams were rejected because of their large weight and possible large expansion properties under changing weather conditions. As with the Panel 6 cameras, the Panel 2 cameras were enclosed in nitrogen purged gas bags. Figure 4 shows the setup for ET Panel 2. 


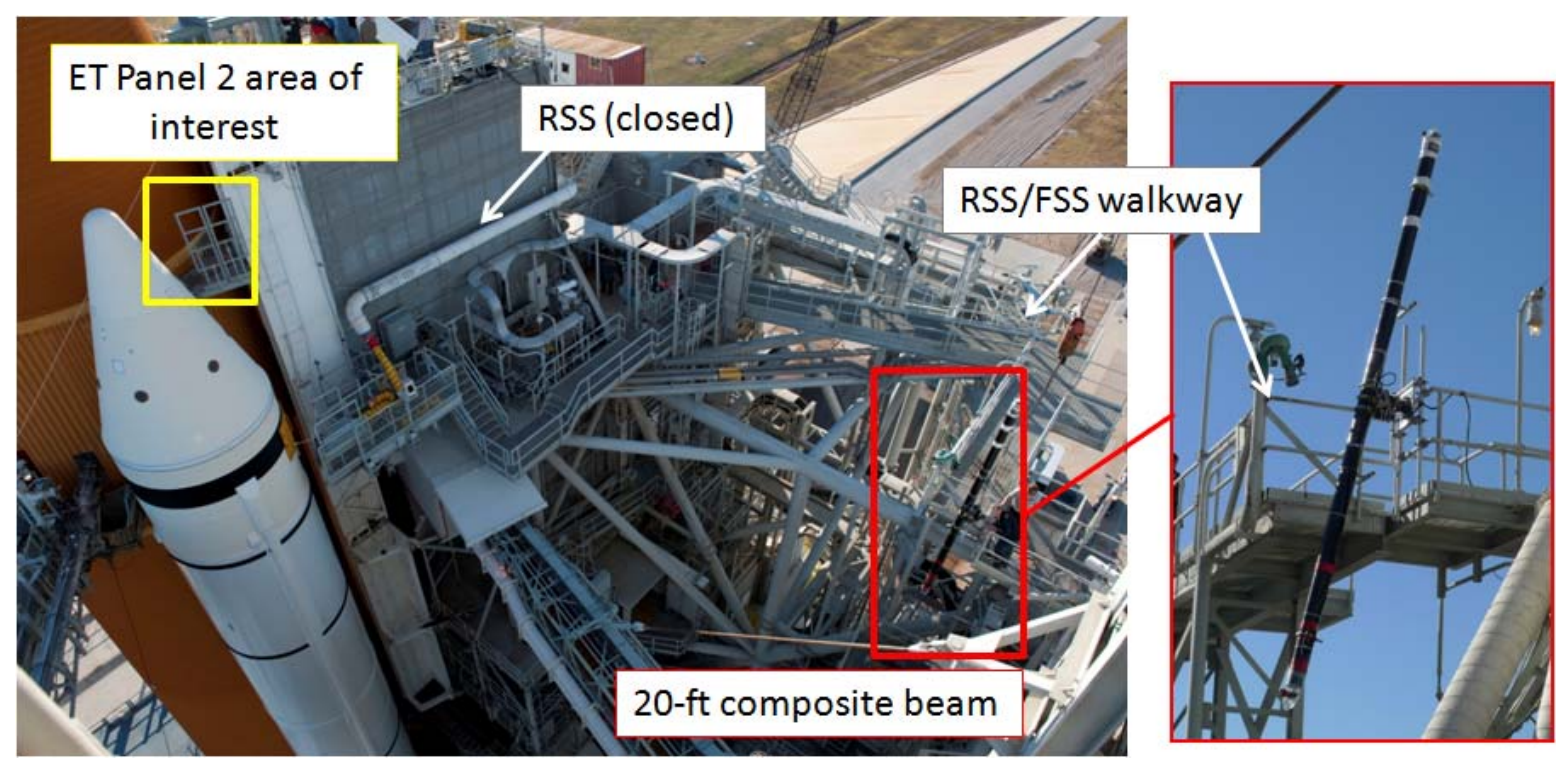

Fig. 4 Details and camera setup for ET Panel 2

\section{Calibration Procedures}

In photogrammetry techniques, calibrations of the cameras are needed prior to the acquisition of the image data. Calibration procedures are similar for all types of photogrammetric imaging, which identify and correct for camera lens distortion, establish absolute scaling, and determine the relative positions of each camera in space or in relation to the other cameras. The calibration procedure also establishes a virtual box, inside of which all measurements acquired are considered valid. Calibrations for both sets of cameras were performed on the base of Launch Pad 39-A prior to hoisting the stereo camera rigs to the FSS. The calibration procedure required that each camera pair acquire images of a calibration object through a sequence of rotations, angles, and, finally, repositions at various distances ranging from $\pm 10 \mathrm{ft}$ to the camera pair. The calibration object was a large 2 meter cross covered with patterns capable of being recognized by the image correlation calibration software. Figure 5 shows the calibration object, mounted on a tripod on the ground of Launch Pad 39-A.

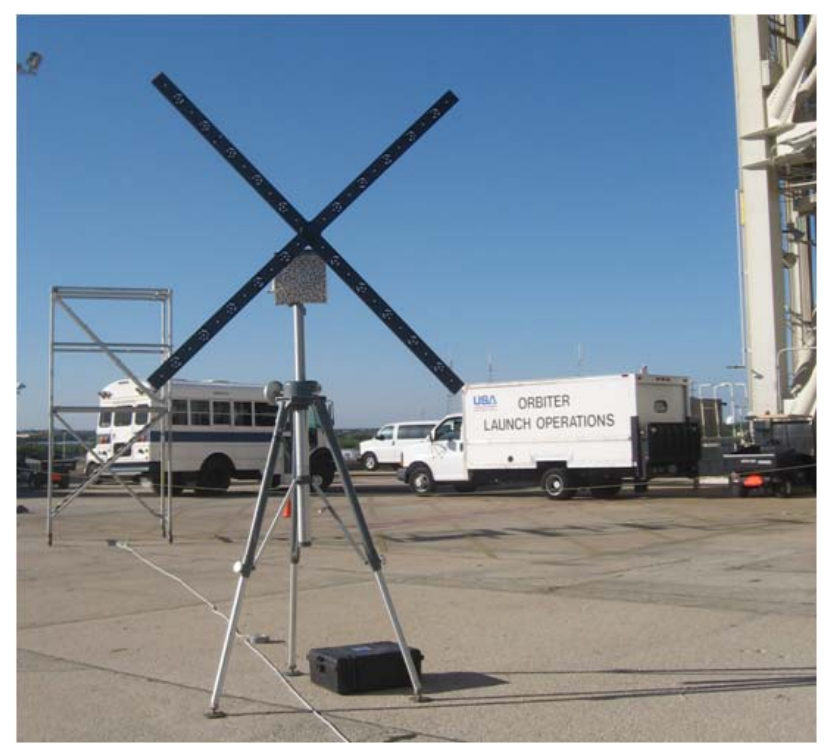

Fig. 5 Calibration object 
Ground calibration was chosen because many of the repositions and rotations needed during the calibration procedure would be inaccessible or unsafe if attempted at each pair's final location on the FSS. However, the calibration integrity could be compromised if either the hoisting process or the thermal expansion of the camera beam caused large relative movement and/or change in angle within each pair of cameras. These risks were deemed acceptable and outweighed the risks of the alternative calibration procedure in the camera final mounted position.

\section{Speckle Application Methodology}

A dot speckle pattern was stenciled on the ET Panel 2 and Panel 6 locations by orbiter technicians with approved black epoxy marker paint. The dots were nominal 3/8-in diameter, and they were painted between stringers 5 and 14 on Panel 6 and over the repaired region for Panel 2. For Panel 6 , the pattern extended from just above the LO2 flange, down onto the intertank structure, and approximated a T shape. For Panel 2, the pattern resembled a square painted over the repaired area shown in Figure 2. Figure 6 shows a close up view of the application of the dot speckle pattern on Panel 6.

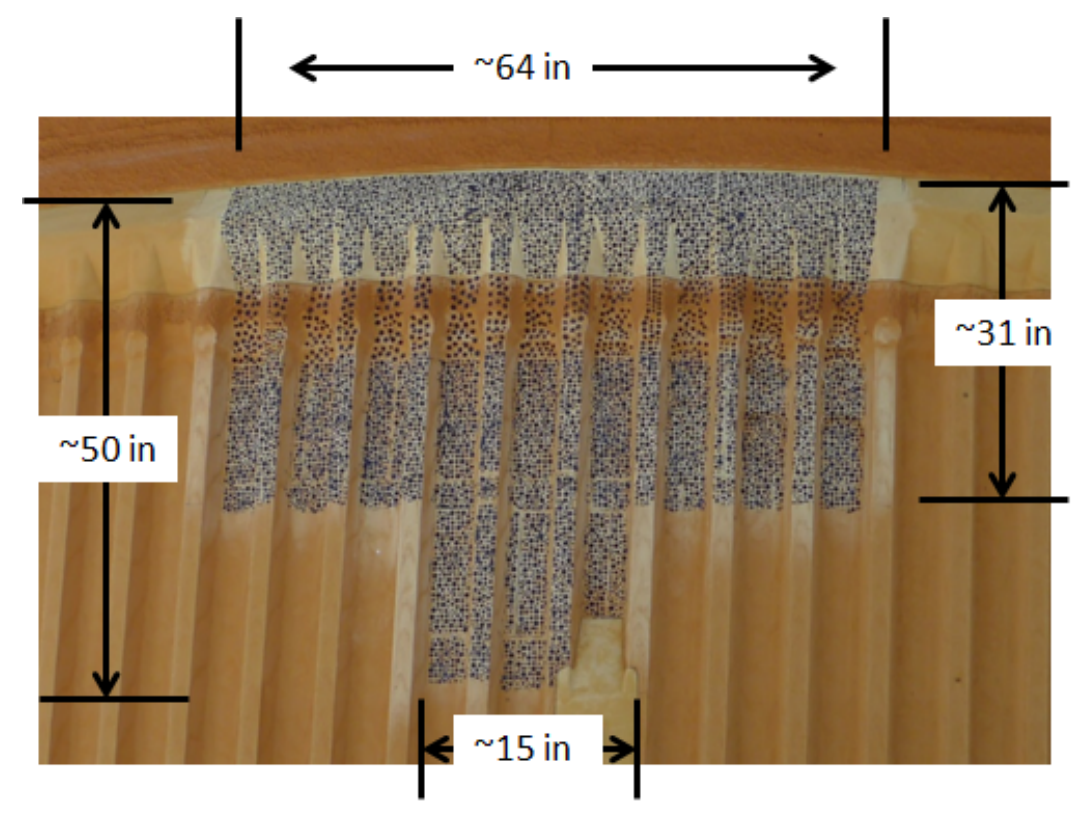

Fig. 6 Speckle pattern on ET Panel 6

\section{Image Acquisition Procedures}

Both camera pairs were controlled from the Launch Control Complex (LCC) Firing Room 3, 3.5 miles away, via underground fiber optic cable. The Panel 2 cameras were connected into and directly controlled by the photogrammetry rack-mounted computer, which physically stayed on the FSS walkway during the tanking test. The photogrammetry rack-mounted computer was controlled in the LCC by a remote desktop connection. Each individual Panel 6 Phantom camera was controlled via its own dedicated computer in the LCC. A camera trigger signal originated from the photogrammetry rack computer and was sent to all cameras, and a common IRIG time code originating from LCC was input into each camera for synchronization purposes. The results were pairs of synchronized uncompressed black and white TIFF images. A schematic of the setup is shown in Figure 7. 


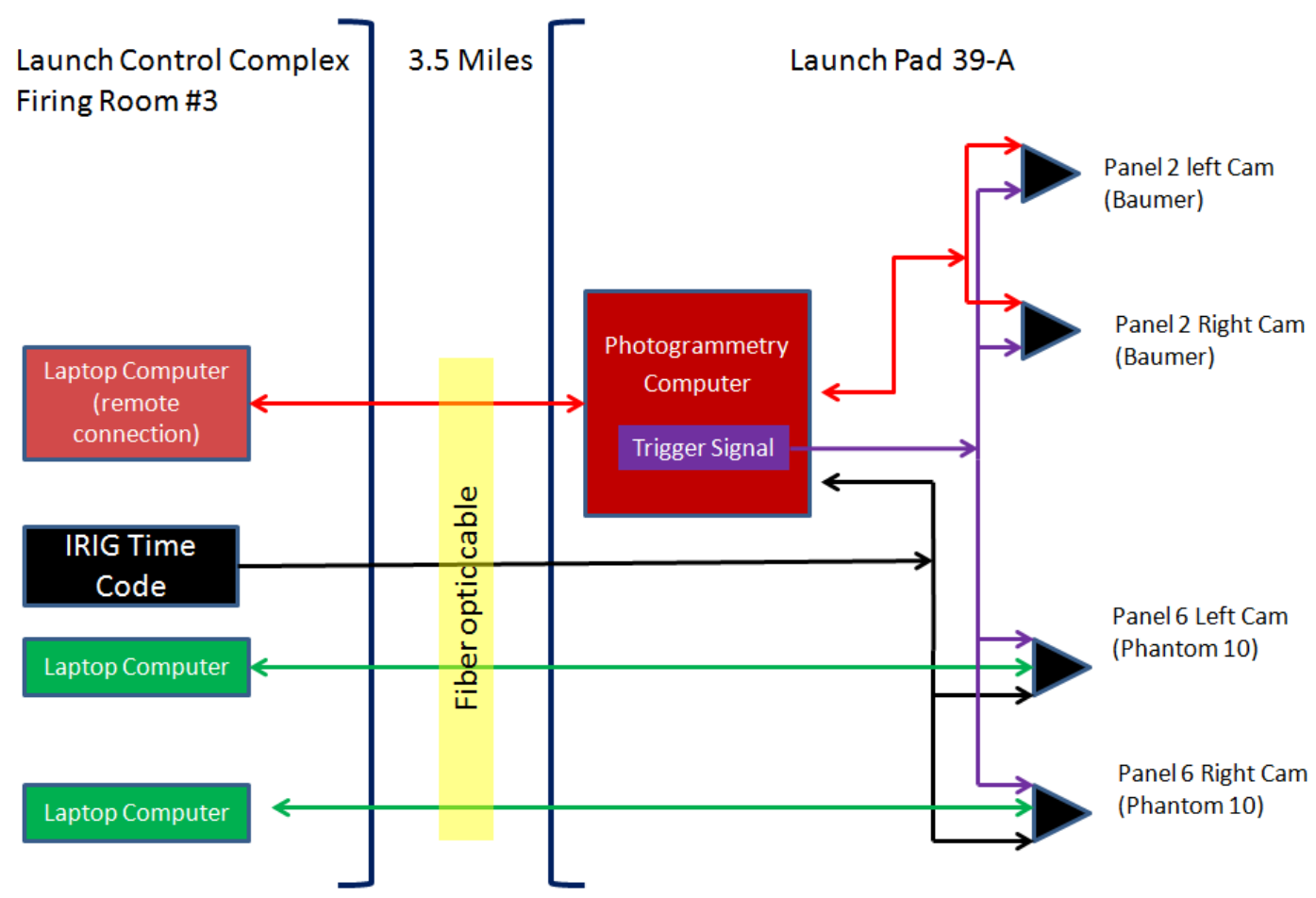

Fig. 7 Photogrammetry setup schematic

\section{Results}

There were 1,637 images, spanning the entire duration of the test, successfully acquired from each camera and used for photogrammetric analysis. The tanking test started at approximately 7:19 am KSC local time and lasted approximately 7.5 hours (from first fill to final drain). Image acquisition ended one-half hour after the 5 percent tank sensor indicated dry conditions were present. The extra half hour was included to ensure that the ET was empty of propellant and returned back to its pre test, empty state. The general acquisition rate was every 10 seconds during the fill, every 3 seconds during the pre-pressurization (prepress), and every 60 seconds during the drain. A test schedule highlighting significant test events is shown in Table 1.

Table 1 Significant events noted in the tanking test

\begin{tabular}{|l|l|l|l|}
\hline & & IRIG/ EST (local KSC & \\
\cline { 3 - 4 } Image \# & GMT & time) & Event \\
\hline 0 & $12: 19: 38$ & $7: 19: 38$ & Test begin \\
\hline 112 & $12: 38: 27$ & $7: 38: 27$ & LO2 Slow Fill Began \\
\hline 202 & $12: 53: 50$ & $7: 53: 50$ & LO2 5\% Sensor Wet \\
\hline 313 & $13: 12: 34$ & $8: 12: 34$ & Equivalent Time of Stringer Crack \\
\hline 900 & $14: 52: 25$ & $9: 52: 25$ & LO2 Fast Fill Ended \\
\hline 1245 & $19: 18: 35$ & $2: 18: 35$ & LO2 Pre-pressurization (prepress) Began \\
\hline 1306 & $19: 24: 44$ & $2: 24: 44$ & LO2 Prepress Ended \\
\hline 1465 & $19: 51: 49$ & $2: 51: 49$ & LO2 Drain Began \\
\hline 1607 & $21: 21: 05$ & $4: 21: 05$ & LO2 5\% Sensor Dry \\
\hline 1637 & $21: 51: 31$ & $4: 51: 31$ & End of data acquisition \\
\hline
\end{tabular}


All results were examined post-tanking test. When examining the results post-test, it was noted that Panel 6 data were of much higher fidelity than Panel 2 results and will therefore be used as the example for the data analysis. Figure 8 shows sample images captured from the left and right camera of Panel 6. The calibrated region is much larger than the patterned region based on the original goal of measuring a significant portion of the length of the intertank. However, the patterned region was significantly reduced due to flight safety concerns.

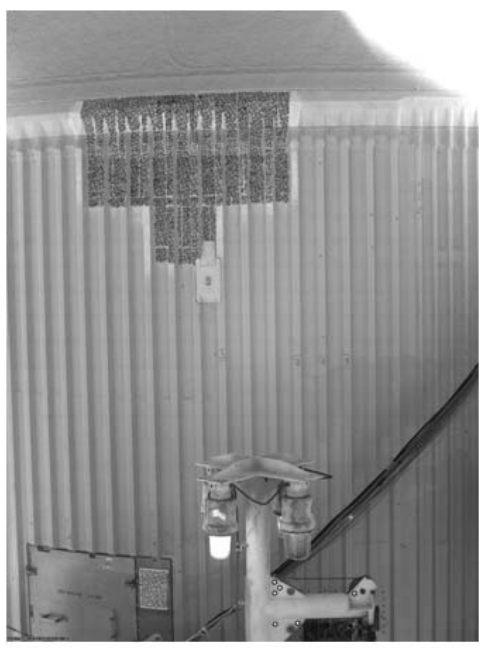

Left Camera

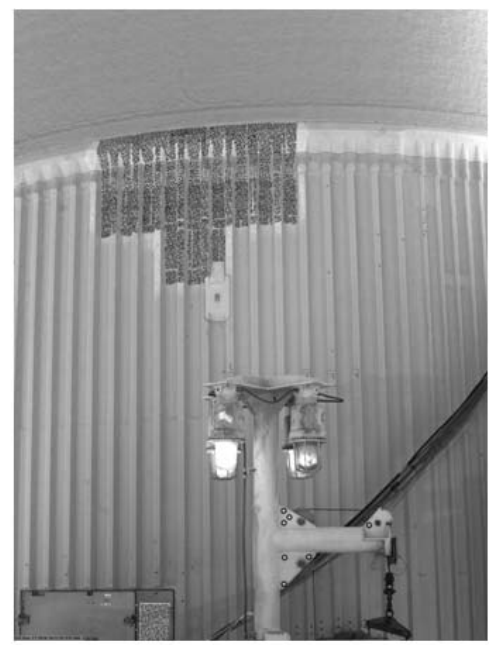

Right Camera

Fig. 8 Panel 6 stereo camera view

The data were first used in a qualitative surface analysis examination. The surface analysis was a three dimensional surface visualization feature. It was used as a quick look to determine whether any anomalies (i.e., stringer cracks) occurred on the ET surface. All nominal conditions were detected from the surface analysis. Several features including the radial curvature of the ET, the peaks and valleys of the stringers, and the LO2 flange were highly noticeable. Figure 9 shows the surface analysis of the ET, visualized in blue. The horizontal lines seen in Figure 9 will be addressed later in this section.

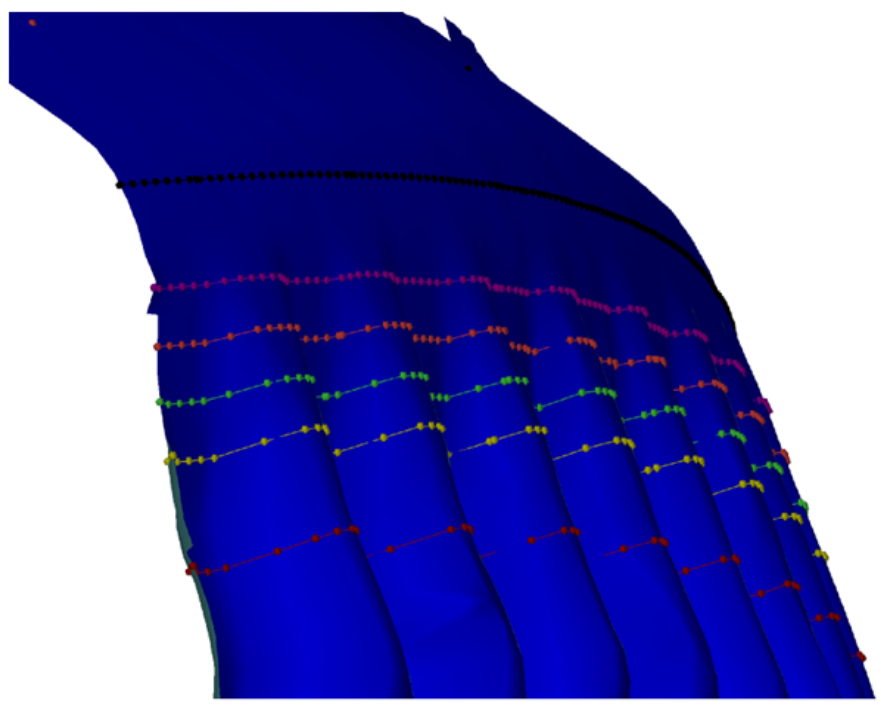

Fig. 9 Surface analysis visualization

Quantitative measurements were next examined. The data density contained over nine data points per square inch, with over 20,000 virtual strain gages available from which to make the quantitative measurements. A coordinate system was 
created such that the $+x$ direction was defined as sideways to the right in the images, representing the ET hoop direction, and the $+y$ direction was defined as vertical toward the top of the ET, representing the axial direction. Finally, the $+z$ direction was defined as outward from the tank, representing the radial direction.

Figure 10 shows two ways of extracting the data from the full field measurements. The left image shows a fringe plot of the unmodified full field radial (z) displacement just at the completion of fill (image 900). The change in radial displacement is very noticeable and indicates significant movement in the ET, with an inward motion of more than 0.5 inches at the top of the flange. Gaps or holes in the data indicate portions of the pattern that were not tracked and were mainly due to the discontinuity of the dot pattern resulting from the abrupt change in direction between the stringer tops and valleys. From the fringe plots, particular places of interest were identified for further time history analysis. Figure 10, right, shows locations of four discrete points identified for further analysis.

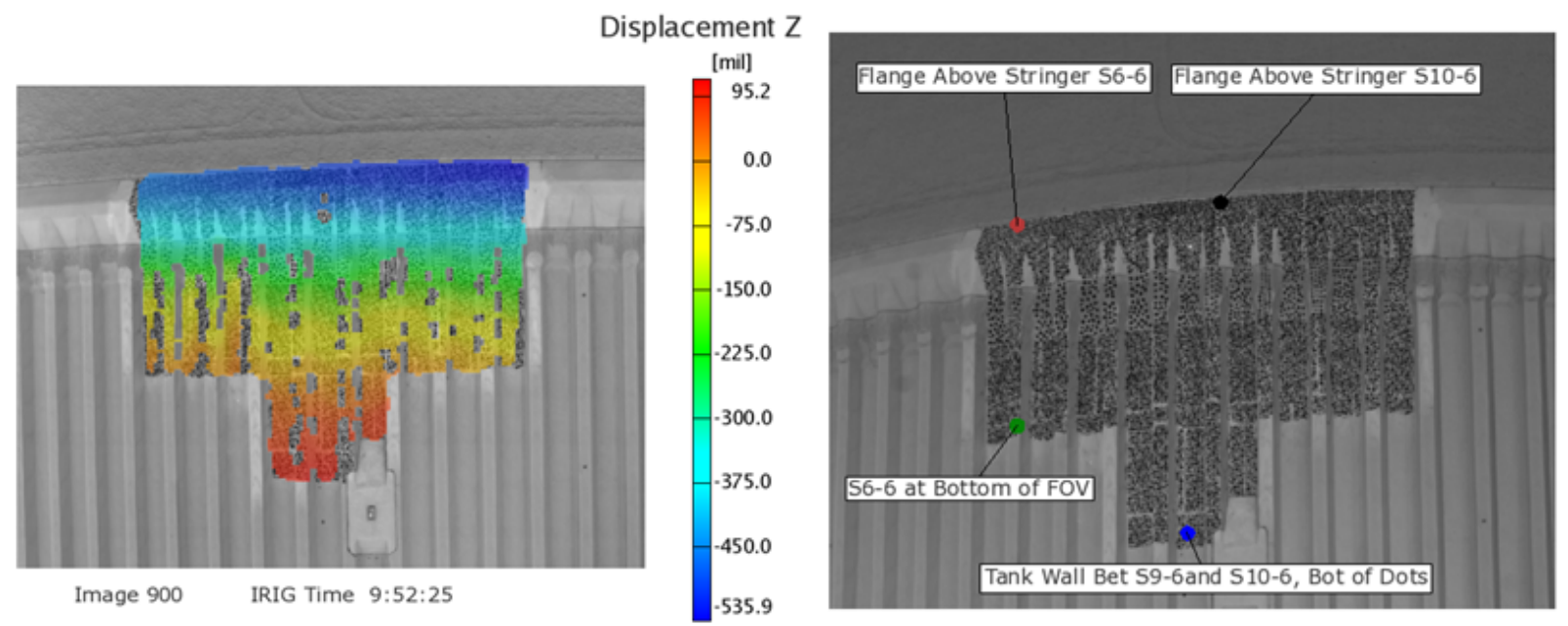

Fig. 10 Full Field fringe plot (left) extracted points for time history analysis (right)

An interrogation of the time history was performed on the point labeled in Figure 10 as "Flange above stringer S6-6", which is located near the upper left of the pattern. Figure 11 shows the time histories of the three dimensional motion at this point. Note that "disp e" represents the root-sum-square of the motion in three directions at this location. Significant event milestones are labeled above the data for reference. 


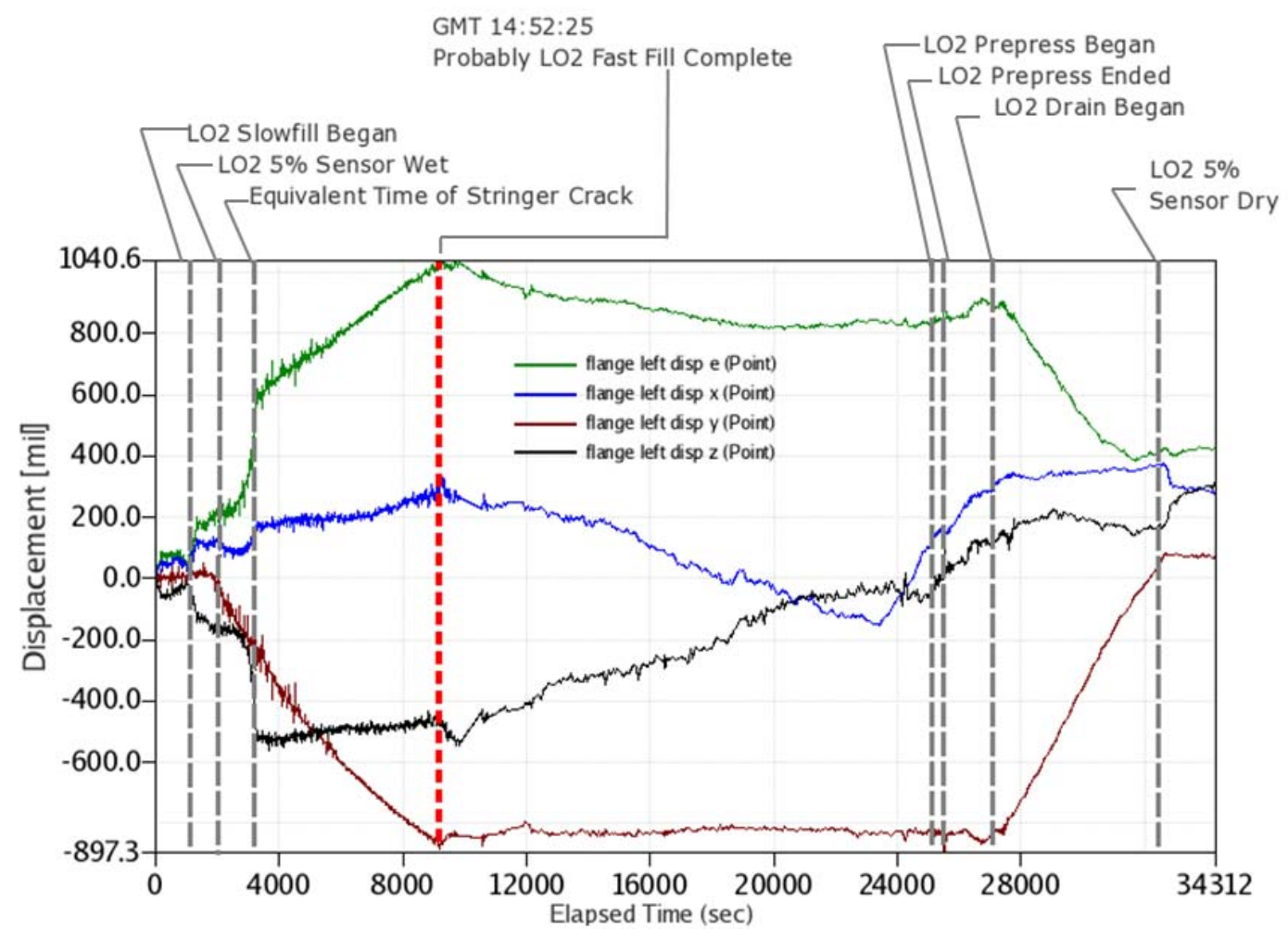

Fig. 11 Time history of displacements at a point on the left flange

The time history shows that between the LO2 slow-fill milestone and the completed LO2 fast-fill milestone, the point on the left flange exhibited almost 900 mils of vertical displacement in the $-y$ (downward) direction, which is represented by the plateau in this line in Figure 11. This displacement disappears at the milestone when the LO2 drain began, and the point reaches almost the starting position after the LO2 sensor dry milestone. This finding is due to the sag in the ET from the weight of the propellants. Also, the radial displacement, which is represented by the black line in Figure 11, is negative, indicating contraction due to the super-cooled liquid inside the ET. Finally, the horizontal displacement, represented by the blue line in Figure 11, is positive, indicating displacement toward the right of the image.

An examination into the relative deformation between stringer peaks and valleys was next conducted. The differential motion function was used, with two endpoints that were located on a stringer peak and a stringer valley. The differential motion of a valley between stringers 11-12 (S11-12) and stringer 13 (S13) were compared in Figure 12. 

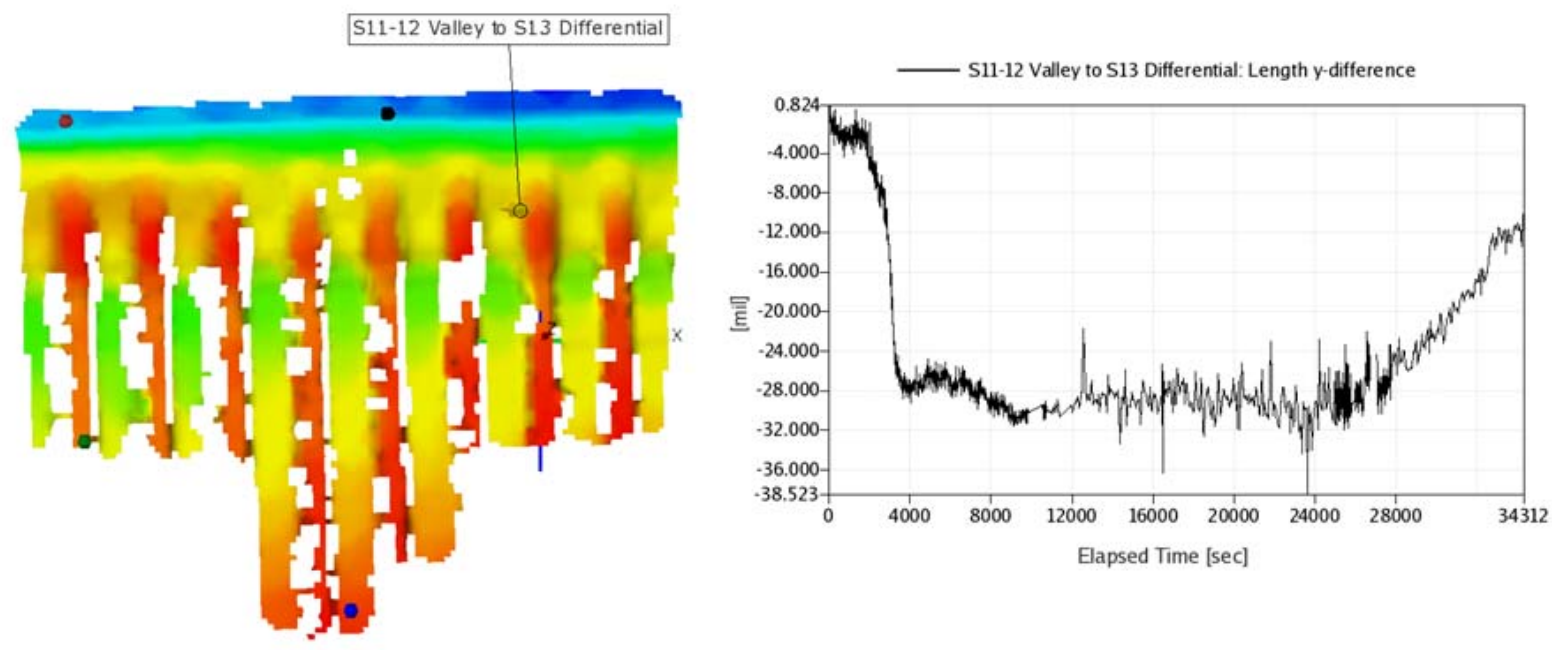

Fig. 12 Analysis for stringer crack determination

The plot in Figure 12, right, shows the vertical difference between the stringer peak and valley. A large abrupt spike represented by a discontinuity in the data would indicate a possible crack formation, as a crack would show significant shearing motion between the peak and the valley. In contrast, the gradual changes seen over the course of the first 4000 seconds of the test were determined to be normal ET motion. Similar analyses were conducted for the other stringer locations. The analysis of this data did not show evidence of any newly developed cracks that might have occurred during the tanking test.

Spatial trends in the data were next extracted using the section line function. A section line represented the data as a function of position for a discrete time, rather than plotting as a function of time. The section line in Figure 13 (shown in red) extended vertically between the top and bottom of the speckle region in a valley between stringers 8 and 9 .

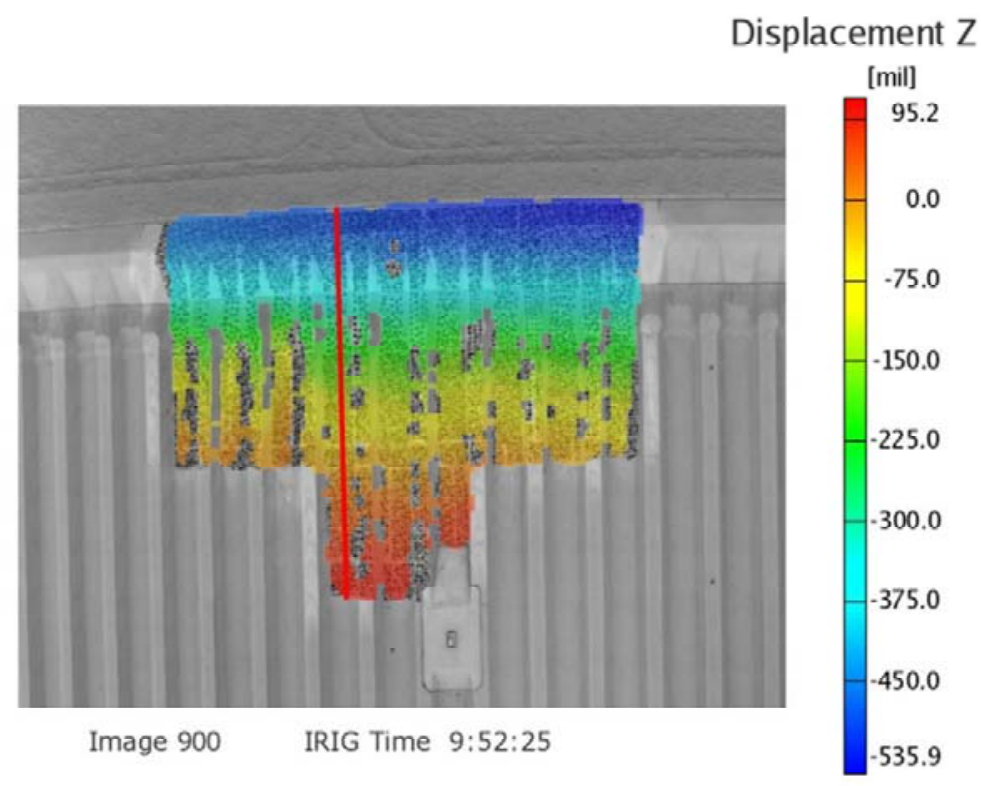

Fig. 13 An extracted section line for the valley between stringers 8 and 9 
The data were acquired for significant times of interest that occurred throughout the test, mainly those that are referenced in Table 1. Relative motion between these significant times was determined by the difference in the sequential section line data. For example, the difference in radial displacement between LO2 Sensor Wet (image 202 data) and LO2 Slow Fill Begin (image 112 data) was found by subtracting the data set from image 112 from the data set in image 202 . The result is shown as the blue line labeled "202-112" in Figure 14, below. Rigid body motion, otherwise known as motion that occurred on the entire ET, resulted in the lines showing a flat, horizontal trend. However, a large slope or a large difference as a function of position would indicate relative deformation between the two event times. In theory, the "flatness" of the line determined the type of motion. Figure 14 shows a differential section line analysis.

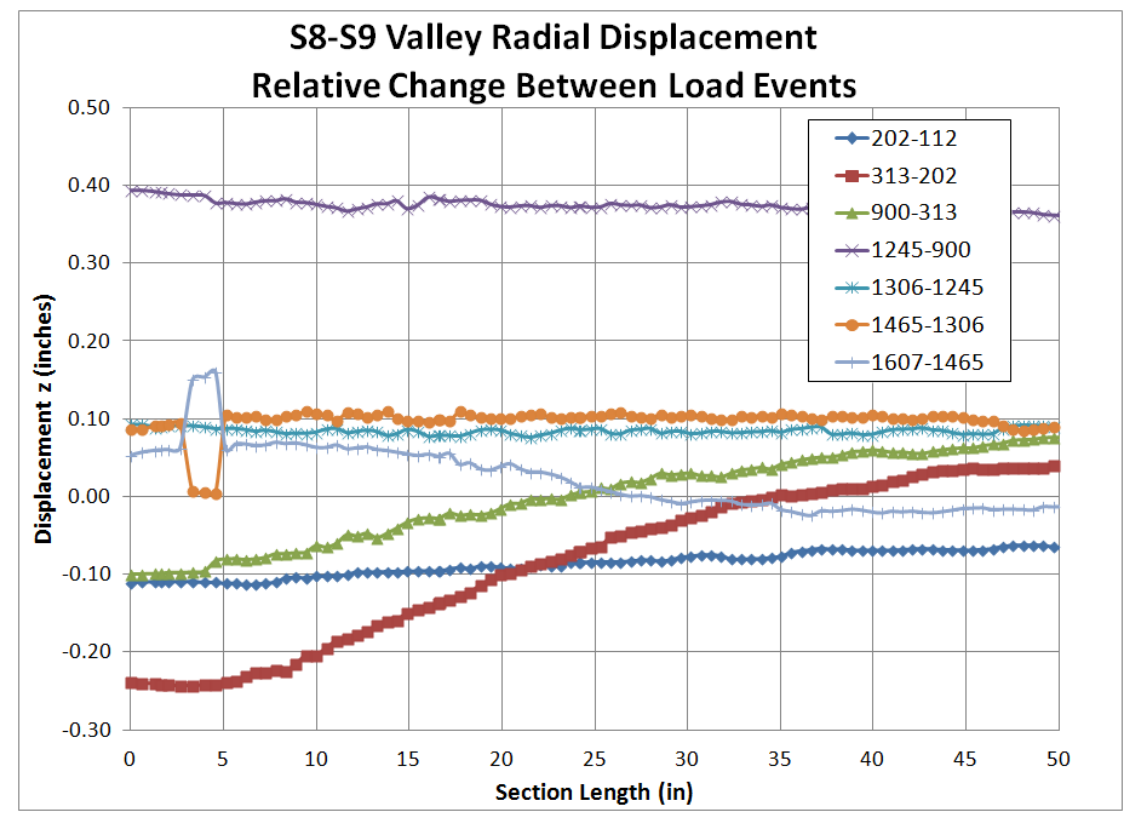

Fig. 14 Relative change between significant events for the S8-S9 valley section line

Figure 14 suggests that all events up through the filling of the ET (image 900) cause actual deformation, which is noted by the slope increase in the three data sets leading up to image 900. Data set "202-112" shown in blue in Figure 14, starts off as a flat line indicating rigid body motion. However data set "313-202" shown in maroon in Figure 14, shows a significant change in radial ( $\mathrm{z}$ ) displacement throughout the section length, indicating relative motion. Data set "900-313", shown in light green in Figure 14, shows a similar trend. The difference between LO2 fast fill and LO2 pre-pressurization (images 1245 and 900, or time $25559 \mathrm{sec}$ and $9590 \mathrm{sec}$ ) seems to cause rigid body motion only. Similar phenomena can be seen between the difference of the LO2 drain (image 1465) and the end of the LO2 prepress (image 1306). The remaining curves show some form of sloped line and most likely indicate structural deformations of the ET. The minor plateau around the 5 inch mark in the data represents a false reading in the image 1465 data due to data dropout.

A complete analysis which includes further section line extraction and data analysis was performed by NASA Marshall Space Flight Center (MSFC). Because stringer cracks were not seen in the Panel 6 data, the majority of the data was used to compare and validate computer modeling efforts on the ET performance. Figure 15 shows a comparison of photogrammetry data, shown in solid lines, to finite element analysis predictions for flange radial deflection and rotation angle. For a complete analysis on the test-simulation correlation, refer to reference 17. 

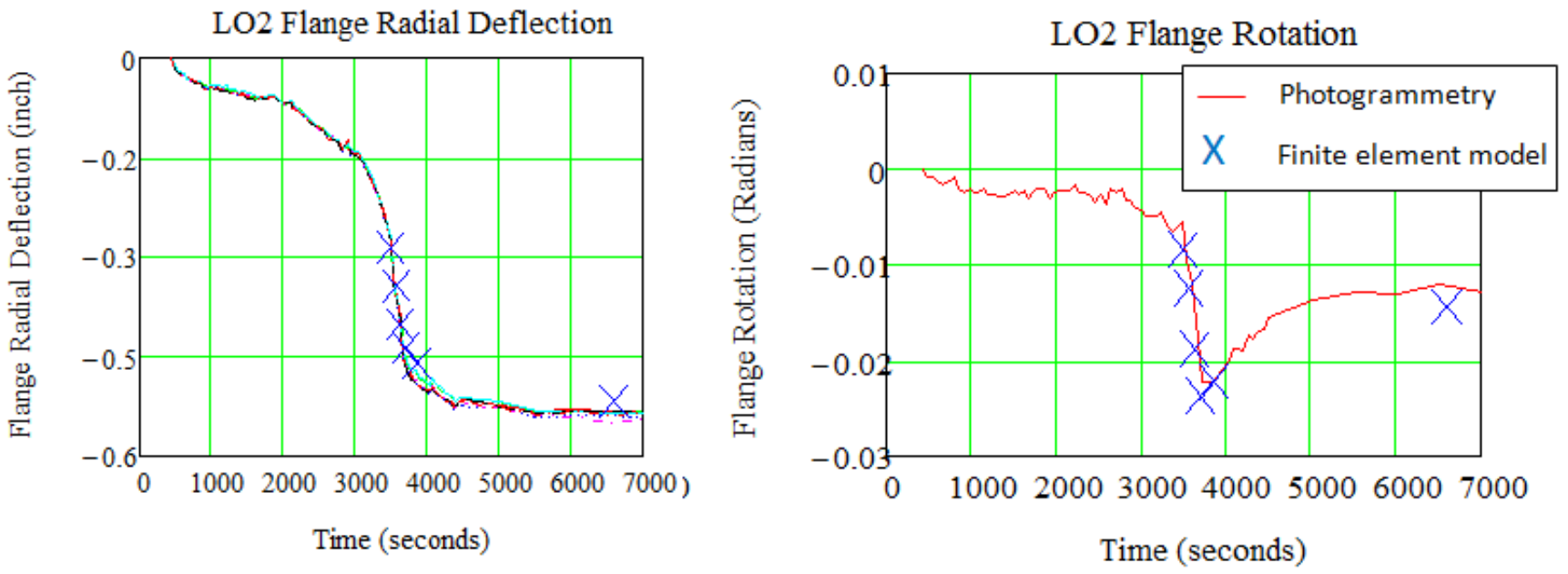

Fig. 15 Comparison of photogrammetry data to finite element analysis

\section{Summary}

On December 17, 2010, three dimensional digital image correlation techniques were successfully used as a part of a Space Shuttle tanking test to help determine root causes for stringer cracks seen on ET-137 in support of the STS-133 mission. The setup and challenges, along with the final methodology used and some sample results are presented.

Photogrammetric engineers had 10 days from the first definition of the problem to the day of the tanking test. In this span of time, they had to formulate a plan of action, conduct a site visit to familiarize themselves with the Space Shuttle and Launch Pad 39-A launch hardware, scout suitable locations for camera placement, order and fabricate materials and components, ship equipment, organize support, calibrate equipment, mount equipment and acquire test images. All of these items were completed on a non-interference basis, without compromising the safety of personnel and hardware or the integrity of the data. It was the first time that stereo photogrammetric imaging equipment had been used on the launch pad and the first time that measurements had been acquired on the ET during a tanking test.

During the tanking test, photogrammetric engineers were located in the Launch Control Center's Firing Room 3, 3.5 miles away from Launch Pad 39-A. They were remotely controlling cameras housed in explosion proof housings mounted on custom fabricated camera bars located on the 215-ft level of FSS at Launch Pad 39-A. Approximately 1,600 images were successfully acquired during the 8 hour test.

From the measurements, the Panel 6 detailed analyses provided significant insight into tank behavior. The images were computed post-test and the resulting data was in the form of full field fringe plots, data extraction time history analysis, section line spatial analyses and differential peak-valley motion. The results show clear deformation in the ET during the test, especially during first fill. The measurements acquired also provided an excellent opportunity to validate computer modeling efforts. The data was used in a complete data analysis and computer modeling effort by MSFC and yielded excellent results which validated the stringer finite element analysis.

ET-137 carried Discovery for the STS-133 mission to orbit on February 24, 2011. 


\section{References}

[1] NASA. http://www.nasa.gov/mission_pages/shuttle/shuttlemissions/sts133/main/index.html. Accessed January 10, 2012.

[2] Harwood, W. "NASA Zeros in on Root Cause of Shuttle Tank Cracks". http://spaceflightnow.com/shuttle/sts133/110111rootcause/. Accessed May 9, 2011.

[3] GOM Optical Measurement Techniques. “ARAMIS User Manual - Software v 6.1”. April 20, 2009.

[4] Schmidt, T et al. "Performance Verification of 3D Image Correlation Using Digital High-Speed Cameras." Proceedings of 2005 SEM Annual Conference, Portland, OR, June 7-9, 2005

[5] Melis M. et al. "Impact Testing on Reinforced Carbon-Carbon Flat Panels with BX-265 and PDL 1034 External Tank Foam for the Space Shuttle Return to Flight Program." NASA TM 2009-213642/REV1, 2009.

[6] Ambur, D. R. et al. "Progressive Failure Studies of Stiffened Panels Subject to Shear Loading." Composite Structures, Vol. 65, pp. 129-142, 2004.

[7] Littell, J.D. et al. "Measurement of Epoxy Resin Tension, Compression and Shear Stress Strain Curves over a Wide Range of Strain Rates Using Small Test Specimens." J. Aerosp. Eng., Vol. 21, pp. 162-173, 2008.

[8] Amsterdam, E. et al. "Failure Mechanisms of Closed Cell Aluminum Foam Under Monotonic and Cyclic Loading." Acta Materialia, Vol. 54, pp. 4465-4472, 2006.

[9] Lesser, W.P et al. “Fatigue Crack Closure Analysis Using Digital Image Correlation.” NASA TM 2010-216695, 2010.

[10] Chevalier, L. et al. "Digital Image Correlation used to Analyze Multiaxial Behavior of Rubber-like Materials." European Journal of Mechanics A/Solids 20, pp. 167-187, 2001.

[11] Revilock, D. M. et al. "Three-Dimensional Digital Image Correlation of a Composite Overwrap Pressure Vessel During Hydrostatic Pressure Tests." NASA TM 2007-214938, 2007.

[12] Thornburgh, R.P and Hilburger M.W. "Longitudinal Weld Land Buckling in Compression Loaded Orthogrid Cylinders." NASA TM 2010-216876, 2010.

[13] Tyson, J. et al. "Biomechanics Deformation and Strain Measurements with 3D Image Correlation Photogrammetry." Experimental Techniques, Vol. 26, Iss. 5, pp. 39-42, 2002.

[14] Zhang, D. et al. "Evaluating the Material Behavior of Arterial Tissue using Digital Image Correlation." Experimental Mechanics, Vol. 42, No. 2, pp. 409-416, 2002.

[15] Chen, J. et al. "Marking-dots Digital Image Correlation and Application to Studies of Spinal Biomechanics." Proc. SPIE, 5852, p. 455, 2005.

[16] Moerman, K. M. et al. "Digital Image Correlation and Finite Element Modeling as a Method to Determine Mechanical Properties of Human Soft Tissue In Vivo." J. of Biomechanics, Vol. 42, pp. 1150-1153, 2009.

[17] Oliver, S.T. "STS-133/ET-137 Tanking Test Photogrammetry Assessment." AIAA / ASME / ASCE / AHS / ASC Structures, Structural Dynamics, and Materials Conference, Honolulu, HI, to be presented April 23-26, 2012. 


\section{Acknowledgments}

Successful completion of these measurements relied on major contributions from dozens of individuals. The authors were very fortunate to be assigned Wulf Eckruth of United Space Alliance as our primary liaison, and were pleased that he was awarded a NASA Silver Snoopy in recognition of his critical efforts. Matthew Van Den Driessche and Gabor Tanacs also provided on-the-fly design engineering assistance. Mike Payne and Jack Strieter (NASA KSC) provided very strong overall engineering supervision and guidance. Mark Olszewski and Robert Stratton of Analex gave photographic equipment and advice on numerous occasions.

Patterning the external tank was a major task in and of itself. Eugene Sweet of Lockheed Martin was the team lead for this effort. Kerry Chreist produced the final design of the dot pattern. JJ Laplante, Tony Marketon, James O'Bryhim and John Hubinger applied the pattern, working on an elevated platform in cold weather until after midnight. Ed Carillion, Scotty Dornton and Timmy Wirth were there to mix the epoxy paint.

The High Crew and Heavy Equipment teams provided extremely strong support numerous times during this effort. High Crew produced custom work and support platforms remarkably quickly. The group included James Gibson, David Schuermann, Tony Pope, Thomas Blabey, Brian Amick, Donald Redfern, Dennis Elmore, Michael Rajnish, Joel Smith, William Evans, Robert Hanover, John Jones, and Mark Kobilis. Heavy Equipment operated trucks and cranes while also demonstrating their own lift capacity in situations where there was only one chance to do the job correctly. Members included Paul Kruse, Wally Majid, Susan Thai, Kevin Hunt, Roger Spillers, David Parker, Andrew Shepard, Philip Reno, James Speigner, David Kobilis, William Mayhew, J. Rhode, Hiawatha Brown, Peter Collins, W. King and Gary Osbourne.

The Launch Equipment Shop was called on for rapid production of many custom components. Gino Digiovanni, Chuck Gardener, Carson Yates, Oogie Townsend, John Franasiak, and John McCauley got the job done effectively and efficiently over multiple shifts.

Dave Sutton, Mike Beville, Abdoul Alivandi and William Sands quickly designed and installed custom nitrogen purge equipment for the cameras to meet safety requirements, without which, installation on Launch Pad 39-A's fixed service structure would not have been permitted.

Tim Wright, Jim Blair and Bruce Hodge were responsible for timing and countdown aspects of the test, including camera communication and triggering support. Gus Alex provided image engineering support.

Brenda Blackmon and Kim Phillips appeared at the launch pad late one evening to install custom thermal blankets on the camera beams, which they had designed and fabricated on short notice. It is unlikely that the sensors would have remained calibrated without this insulation.

The entire effort was heavily documented photographically by IMCS photo and media services. Technical photographers included Tim Terry, Robert Murray, Kenneth Allen, Kevin O'Connell, George Roberts, and Charles Robinson. 